\title{
A Survey of Cloud Computing Architecture and Applications in Health
}

\author{
Carmelo Pino and Roberto Di Salvo \\ Department of Electrical, Electronics and Computer Engineering (DIEEI) \\ University of Catania \\ V.le Andrea Doria, 6 - 95125 (Italy) \\ \{cpino \& disalvo\}@dieei.unict.it
}

\begin{abstract}
The growth of data produced by the medical and clinical community requires the introduction of advanced techniques and resources in terms of computational and storage capabilities. The different problems and needs that afflict the information technologies for health support conduce to adoption of new infrastructure and application based on cloud computing. The different problems concerning to one side to the managerial, administrative and management aspects, to the other side concern who works as a physician or researcher, that needs the infrastructure to process, store, manage patient data, analysis, diagnosis, and so on. Today, cloud computing represents an important alternative to ensure high performance data processing and easy management of the complex tools in different area and in health. Cloud computing can solve many of these problems providing several advantages in terms of resource management and computational capabilities. In this paper a survey concerning the current models of health that are switching to solutions based on cloud computing, is proposed. Different applications and services are explored and concluded that the use of cloud computing and in particular of hybrid cloud solution can represent a significant opportunity to increase the development of the health sector in all its aspects.
\end{abstract} Information Technologies;

\section{INTRODUCTION (NEED IN MEDICAL ENVIRONMENT)}

In the recent years, the health has enjoyed the uninterrupted development of new information technologies solutions. This has led to several benefits in terms of new hardware infrastructure, new specific and more sophisticated medical applications, increased speed in data processing, etc., that has allowed to improve and speed up the health services.

The growth of data produced by the medical and clinical community requires the introduction of advanced techniques and resources in terms of computational and storage capabilities. In order to meet the needs of medical departments, hospital must continuously improve the level of modern system by using advanced science and technology innovation.

The new technologies in E-Healthcare are an important part of medical treatment and follow up procedures. The needs in this community ranging from patient registration, appointment with physician, medical prescription and record of clinical diagnostics, to laboratory tests or surgical procedures that are recorded in Electronic Patient Record (EPR) [1] and specific applications. In particular the applications are related to many different kind and producing several types of data and information: specific and experimental applications [16], knowledge discovery [17, 18, 20], orthodontics applications [21, 22], SPECT images analysis [23] and other similar applications that involves new device to capture the patient feedback like eye-tracker [19].

In order to manage and process this heterogeneous amount of data and applications, is important to consider powerful distributed computational and storage technologies.

Grid computing models have been successfully used for high performance computing, data library services and medical grids, data storage etc. [23]. However grid computing could not become a widely acceptable business model primarily due to lack of marketability in non-trustful domains.

Today, Cloud computing represents an essential opportunity to develop applications that ensure high performance data processing and easy management of the different tools in medical environment ensuring a consistency storage capabilities, overcoming the Grid lack. Therefore the use of new technologies and new infrastructure involves several issues ranging from costs, appropriate security of data, to the development of specific applications.

This trend is highlighted by the several existent applications and architecture based on Cloud in health. Cloud computing offers several advantages by allowing users to use infrastructure, platforms and software provided by the cloud providers.

In healthcare, the use of cloud computing have been proposed as a means for maintaining health records, monitoring patients, managing diseases and cares more efficiently and effectively, or collaborating with peers and analyzing data [13, 14, 15].

In this paper a survey about the Cloud solutions used in health environment is treated. Several solutions will be analyzed in order to understand limits and advantages of each one. The reminder of the paper is as follows: in Section two, is explained how the Cloud computing can be integrated in the actual health environment, what benefit and what effort involves.

In Section three is reported a survey about the existing solution in health that uses Cloud, explaining which are the strengths and weaknesses of each solutions. In the last section concluding remarks are given. 


\section{CLOUD INTEGRATION IN HEALTH ENVIRONMENT}

\section{A. Health needs}

With the development of the information technologies (IT) and the architecture service and application, the use of these technologies in health environment has become a crucial point to sustain the requirements of the clinical and medical structures. What are the current needs of clinics and hospitals, in terms of software and computer architecture? What solutions can be considered to resolve some of the existing architectural and structural issues? To understand the health needs in terms of IT, it's possible to divide and distinguish two main aspects of this community. The first is related to managerial and administrative and managements aspects. The second is related to physician or researcher that needs the infrastructure to process, store, manage patient data, analysis, diagnosis, and so on. In the table below you can see a brief summary of what are the current issues involved and the existing solutions.

TABLE 1 ACTUAL HEALTH NEEDS IN IT AND EXISTING

\begin{tabular}{|c|c|}
\hline Needs & Existing Solution \\
\hline $\begin{array}{c}\text { Store large amount of data: e.g. } \\
\text { DICOM Images. }\end{array}$ & $\begin{array}{c}\text { PACS, Distributed Storage, } \\
\text { Storage on GRID. }\end{array}$ \\
\hline $\begin{array}{c}\text { Management of patient's data: } \\
\text { e.g. anamnesis, patologies, } \\
\text { sintoms, medical records. }\end{array}$ & $\begin{array}{c}\text { Federated databases, specific } \\
\text { software for db interrogation. } \\
\text { EHR-EPR. }\end{array}$ \\
\hline $\begin{array}{c}\text { Needs for specific software and } \\
\text { hardware for data processing. }\end{array}$ & $\begin{array}{c}\text { Distributed system, } \\
\text { Private server architecture. }\end{array}$ \\
\hline \multicolumn{2}{c}{$\begin{array}{c}\text { Software and hardware } \\
\text { scalability for data management. }\end{array}$} \\
\hline $\begin{array}{c}\text { Needs to extend the } \\
\text { authorizations and to warrant } \\
\text { security policy. }\end{array}$ \\
\hline
\end{tabular}

The actual solutions show several challenges associated to automation in this environment: heterogeneity of devices, protocols, and programming interfaces; the requirement for flexible, impact-free deployment;

The requirement for easy to configure, easy to manage, scalable and, if possible, self-adjusting systems, and others.

The modern health structures should therefore have architectures that ensure:

- Increased speed in IT operations management, configuration, reconfiguration of infrastructure, applications and services.

- Automation of simple repetitive health specific tasks, freeing those who manage the infrastructure for small and continuous interventions.
- The reduction of errors and resources problem, during the use of each specific health applications.

Besides, who manages the IT infrastructures should ensure the provision of services that meet specific requirements:

- $\quad$ Self-Service: the user (physician or nurse) must be able to request the services (bandwidth, computing power, applications) on your own, without the intervention of infrastructure managers.

- Global Accessibility; the services must be accessible from multiple devices, from more places and at all times ensuring privacy and encryption of sensible data.

- Elasticity: the resources must be able to climb (upwards and downwards) quickly and, in some cases, automatically.

These and many others are the typical characteristics of architecture of Cloud Computing.

\section{B. Cloud computing}

With the term Cloud computing we refers to a collection of technologies that allow, typically in the form of a service offered by a provider to the customer, store or process data (via CPU or software) through the use of hardware/software distributed and virtualized Network.

Represents "the big switch" in Information Technologies, the principle on which IT will travel in the future.

Cloud computing is a model for structuring IT resources that redefines the way to manage our computer systems. The models of the services that may be provided are three:

1) SaaS (Software as a Service): in this model the software are delivered as services.

2) PaaS (Platform as a Service): in this model are provided services that allow you to develop, test and deploy an application.

3) IaaS (Infrastructure as a Service): in this model is provided the entire IT infrastructure: processors, storage, network services, and so on.

Different are the companies that developing software to create and manage Cloud architecture. Three, mainly, are the patterns of distribution:

1) Public Cloud: in this model, Cloud computing services are delivered over the Internet from a service provider to different customers.

2) Private Cloud: the cloud computing services are provided by on intranet or internet from the company to its various units (on-premise).

3) Hybrid Cloud: services are built on hybrid infrastructure that use the private mode for certain aspects (for example the storage of data) and the public mode for other (for example the access interfaces). 
The use of cloud computing platform reduces operating costs and improves efficiency of medical resources between the medical institutions. However Medical institutions have a huge amount of standard devices that cannot communicate with cloud computing interface directly.

\section{CLOUD IN MEDICAL ENVIRONMENT (EXISTING SOLUTION)}

The existing solution about Cloud in health environment involves different aspects. A first categorization about solutions (architecture, applications and services) that's use Cloud computing in health environment can be done by defining which are the application and architecture actually existent. The categorization includes:

\section{A. Medical Images archive solutions in the Cloud}

Data related to medical images, represents the biggest set of data treated in clinical environment. In [1], a prototype of a Image archive based on Cloud architecture (and in particular using Microsoft Windows Azure) was implemented. It includes a DICOM (Digital Imaging and Communications in Medicine) server which handles standard store/query/retrieve requests; a DICOM image indexer that parses the metadata and store them in a SQL Azure database; and a web UI for searching and viewing archived images based on patient and image attributes. In [2] is presented a system called MIFAS (Medical Image File Accessing System) to solve the exchanging, storing and sharing on Medical Images of crossing the different hospitals issues. The aims of the paper were to solve the challenge in Medical Image exchanging, storing and sharing issues of EMR (Electronic Medical Record). In [4] is presented a secure PACS Cloud Gateway to access PACS Cloud archive, which provides a high security level and without cloud's provider dependence (using a private Cloud solution). A study about the security issues involved in data storage and sharing through cloud, is presented in [3]. It highlights the different types of security problems and how their existence can affect the cloud users using the model Security as a Service (SECaaS).

\section{B. Data management in Health care institutions using Cloud Computing solutions}

Similarly to the problems related to the images archive solutions, another set of problems regards the management of clinical data. The processes for patients' data collection require a several attentions in order to collect and analyze the information. In [5], is proposed a solution to automate this process by using "sensors" attached to existing medical equipments that are inter-connected to exchange services. The proposal is based on the concepts of utility computing and wireless sensor networks. The information becomes available in the "cloud" from where it can be processed by expert systems and/or distributed to medical staff. In [6] is presented an E-Healthcare model for ubiquitous services for data acquisition archiving and presentation in Cloud. The management issues and security concerns in cloud domains are addressed by a services architecture proposed. The model includes Wireless Sensor Networks besides communication and storage systems for a typical hospital taking advantage of the Cloud Services Architecture (CSA).

\section{Health support system}

Another crucial problem in health regards the management of the emergency. During emergency case management at the emergency department of a hospital, readily access to portions of past patient data and to prehospital care data enables proper diagnosis and treatment, eliminates the risk for medical and medication errors and streamlines the emergency healthcare process. In [7] is described a cloud-based service-oriented architecture (SOA) for the implementation of an electronic emergency patient record system (E-EPR) that provide functionality for managing (retrieving, transforming, exchanging and storing) emergency case information and patient critical medical information in a distributed and ubiquitous manner that supports several platforms and applications. In [8] is proposed an PHR (Patient Health Records) based EMS (Emergency Medical system) in a cloud computing environment. In [9] a study of healthcare services provided through a telemedicine oriented Emergency Health Support System (EHSS) is examined. The paper compares a system deployed in the cloud versus a co-located environment based on data gathered from the prototype. A prototype of Emergency Health Support System has been developed and the system has been modeled and tested to derive performance statistics of the system's capability.

\section{Specific Application}

Applications for Health in Cloud Computing environment can be categorized in several ways. The creation of a private Cloud computing architecture, allows to implements many applications SaaS. However a rough classification involves:

- Information retrieval (Medical Image retrieval, Clinical Data Retrieval) [2, 10].

- Data processing (bioinformatics applications, data mining, etc.) [12].

- $\quad$ Patient monitoring $[9,10]$.

- $\quad$ Cloud Resource Broker [10, 11].

\section{CONCLUSION AND FUTURE DIRECTION}

In this paper we presented a survey of system and applications in health based on Cloud. The different kind and aspect of this specific community make the Cloud the perfect solution to solve the actual problem and to satisfy the different health needs. Hybrid Cloud solution ensure an efficent and robust solution for each medical and clinical department thanks to the use of between public and private aspect and advantages of the cloud distribution model. The use of such technologies opens the doors to a new age for the health consisting of high computational powerful, efficent data storage, rapid data retrieval and high interoperability. 


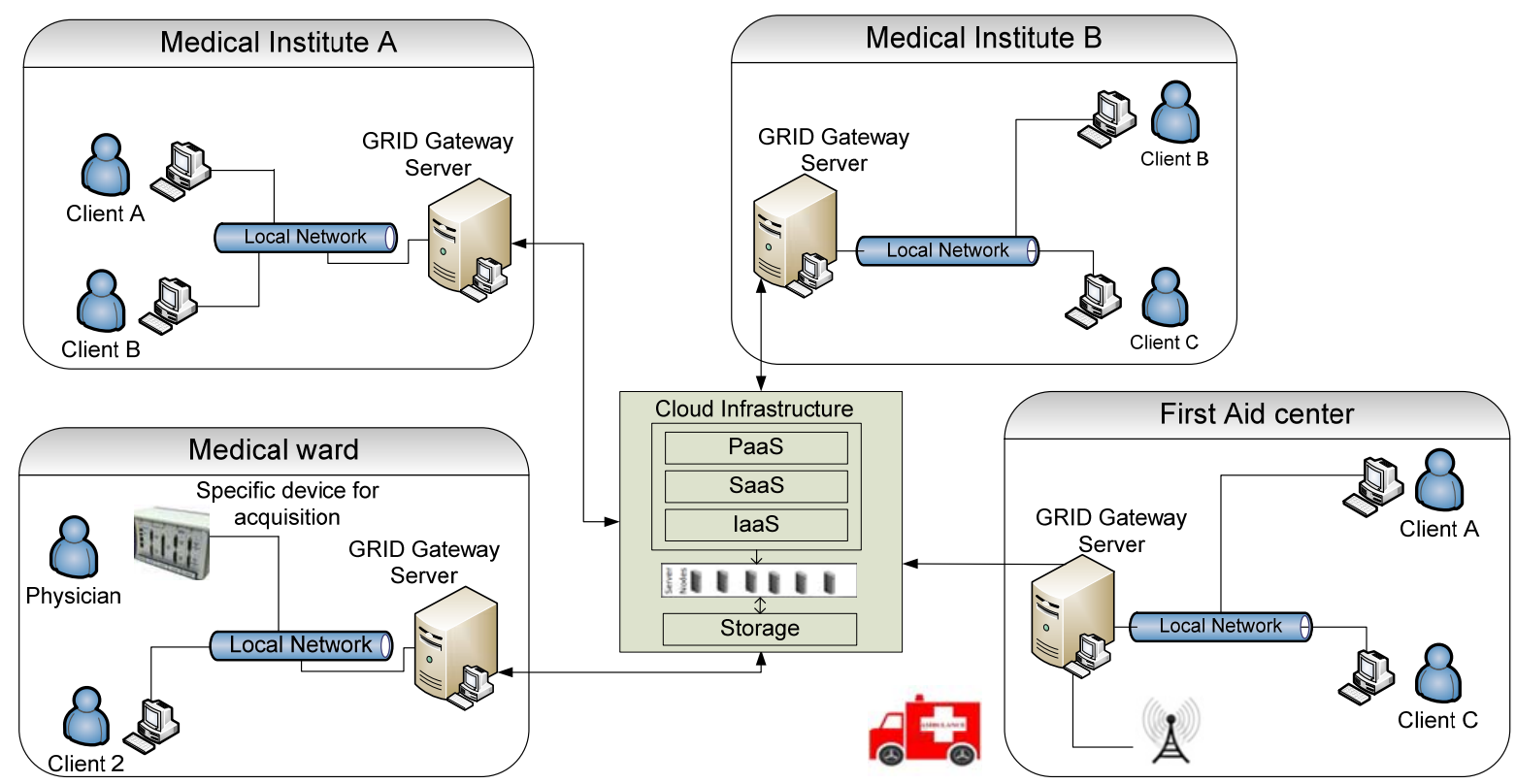

Figure 1. Example of Cloud infrastructure. Different medical institute, first aid center and medical ward can be uses the cloud in order to share and to process data.

\section{REFERENCES}

[1] Chia-Chi Teng, J. Mitchell, C. Walker, A. Swan, C. Davila, D. Howard, T. Needham, "A medical image archive solution in the cloud," Software Engineering and Service Sciences (ICSESS), 2010 IEEE International Conference on , vol., no., pp.431-434, 1618 July 2010

[2] Chao-Tung Yang; Lung-Teng Chen; Wei-Li Chou; Kuan-Chieh Wang; , "Implementation of a Medical Image File Accessing System on Cloud Computing," Computational Science and Engineering (CSE), 2010 IEEE 13th International Conference on , vol., no., pp.321-326, 11-13 Dec. 2010.

[3] S.G. Shini, Tony Thomas, K. Chithraranjan, Cloud Based Medical Image Exchange-Security Challenges, Procedia Engineering, Volume 38, 2012, Pages 3454-3461, ISSN 1877-7058, 10.1016/j.proeng.2012.06.399. 2012.

[4] L.A. Bastiao Silva, C. Costa, A. Silva, J.L.Oliveira, "A PACS Gateway to the Cloud," Information Systems and Technologies (CISTI), 2011 6th Iberian Conference on , vol., no., pp.1-6, 15-18 June 2011.

[5] C.O. Rolim, F.L. Koch, C.B. Westphall, J. Werner, A. Fracalossi, G.S. Salvador, "A Cloud Computing Solution for Patient's Data Collection in Health Care Institutions," eHealth, Telemedicine, and Social Medicine, 2010. ETELEMED '10. Second International Conference on , vol., no., pp.95-99, 10-16 Feb. 2010.

[6] M. Poulymenopoulou, F. Malamateniou, and G. Vassilacopoulos. 2011. E-EPR: a cloud-based architecture of an electronic emergency patient record. In Proceedings of the 4th International Conference on PErvasive Technologies Related to Assistive Environments (PETRA '11). ACM, New York, NY, USA, , Article 35, 7 pages, 2011.

[7] V, Koufi, F. Malamateniou, G. Vassilacopoulos, "Ubiquitous access to cloud emergency medical services," Information Technology and Applications in Biomedicine (ITAB), 2010 10th
IEEE International Conference on , vol., no., pp.1-4, 3-5 Nov. 2010

[8] Yu, D. Weider D. R. Bhagwat, "Modeling Emergency and Telemedicine Heath Support System: A Service Oriented Architecture Approach Using Cloud Computing.." IJEHMC 2 , no. 3 (2011): 63-88, 2011.

[9] S. Mohapatra, K. Smruti Rekha, Sensor-Cloud: A Hybrid framework for remote patient monitoring. International Journal of Computer Applications (0975 - 8887), Volume 55- No.2, October 2012

[10] M.I.B Nordin,. M.I. Hassan, "Cloud resource broker in the optimization of medical image retrieval system: A proposed goalbased request in medical application," National Postgraduate Conference (NPC), 2011 , vol., no., pp.1-5, 19-20 Sept. 2011

[11] Lejiang Guo; Fangxin Chen; Li Chen; Xiao Tang; , "The building of cloud computing environment for e-health," E-Health Networking, Digital Ecosystems and Technologies (EDT), 2010 International Conference on , vol.1, no., pp.89-92, 17-18 April 2010.

[12] A. Rosenthal, P. Mork, M. Li, J. Stanford, D. Koester, P. Reynolds, Cloud computing: A new business paradigm for biomedical information sharing. J. Biomed. Inform. 43, 342-353, 2010.

[13] Wagener, J., Spjuth, O., Willighagen, E. and Wikberg, J. 2009. XMPP for cloud computing in bioinformatics supporting discovery and invocation of asynchronous web services. BMC Bioinformatics. 10, 279, 2009.

[14] R. Zhang, and L. Liu L. Security models and requirements for healthcare application clouds. In Proceedings of 3rd International Conference on Cloud Computing 2010. IEEE Cloud '10, Miami, Florida, USA, 268-275, 2010.

[15] D. Giordano, C. Spampinato, G. Scarciofalo, R. Leonardi, An automatic system for skeletal bone age measurement by robust processing of carpal and epiphysial/metaphysial bones, IEEE 
Transactions on Instrumentation and Measurement, (10) 25392553, 2010.

[16] A. Faro, D. Giordano, C. Spampinato, Combining literature text mining with microarray data: Advances for system biology modeling, Briefings in Bioinformatics, (1) 61-82, 2012.

[17] A. Faro, D. Giordano, F. Maiorana, C. Spampinato, Discovering genes-diseases associations from specialized literature using the grid, IEEE Transactions on Information Technology in Biomedicine, (4) 554-560, 2009

[18] A. Faro, D. Giordano, C. Pino, C. Spampinato, Visual attention for implicit relevance feedback in a content based image retrieval, Eye Tracking Research and Applications Symposium (ETRA), 7376, 2010.

[19] A. Faro, D. Giordano, Design memories as evolutionary systems socio-technical architecture and genetics, Proceedings of the IEEE International Conference on Systems, Man and Cybernetics, 43344339, 2003.

[20] D. Giordano, R. Leonardi, F. Maiorana, G.Cristaldi, M.L Distefano, Automatic landmarking of cephalograms by cellular neural networks, Lecture Notes in Computer Science (including subseries Lecture Notes in Artificial Intelligence and Lecture Notes in Bioinformatics), 3581, LNAI, 333-342, 2005.

[21] R. Leonardi, D. Giordano, F. Maiorana, An evaluation of cellular neural networks for the automatic identification of cephalometric landmarks on digital images, Journal of Biomedicine and Biotechnology, art. no. 717102, 2009.

[22] A. Faro, D. Giordano, C. Spampinato, S. Ullo, A. Di Stefano, Basal ganglia activity measurement by automatic 3-D striatum segmentation in SPECT images, IEEE Transactions on Instrumentation and Measurement, (10) 3269-3280, 2011.

[23] D. Giordano, C. Pino, C. Spampinato, M. Fargetta, A. Di Stefano. Nuclear Medicine Image Management System for Storage and Sharing by using Grid Services and Semantic Web. HEALTHINF, page 80-86. SciTePress, 2011. 